\title{
Laparoscopic Treatment of Gastric Duplication in a Child
}

\section{Barbara Catellani, MD}

Department of Surgery, University of Modena and Reggio Emilia-Policlinico of Modena, Modena, Italy.

\section{Francesca Pecchini, MD}

Department of Surgery, University of Modena and Reggio Emilia—Policlinico of Modena, Modena, Italy.

\section{Lorena Sorrentino, MD}

Department of Surgery, University of Modena and Reggio Emilia-Policlinico of Modena, Modena, Italy.

\section{Francesca Cabry, MD}

Department of Surgery, University of Modena and Reggio Emilia-Policlinico of Modena, Modena, Italy.

\section{Diego Biondini, MD}

Department of Pediatric Surgery, University of Modena and Reggio Emilia—Policlinico of Modena, Modena, Italy.

\section{Pier Luca Ceccarelli, MD}

Department of Pediatric Surgery, University of Modena and Reggio Emilia-Policlinico of Modena, Modena, Italy.

\section{Roberta Gelmini, MD, PhD}

Department of Surgery, University of Modena and Reggio Emilia-Policlinico of Modena, Modena, Italy. E-mail: roberta.gelmini@unimore.it

(c) Mary Ann Liebert, Inc. DOI: 10.1089/vor.2017.0451
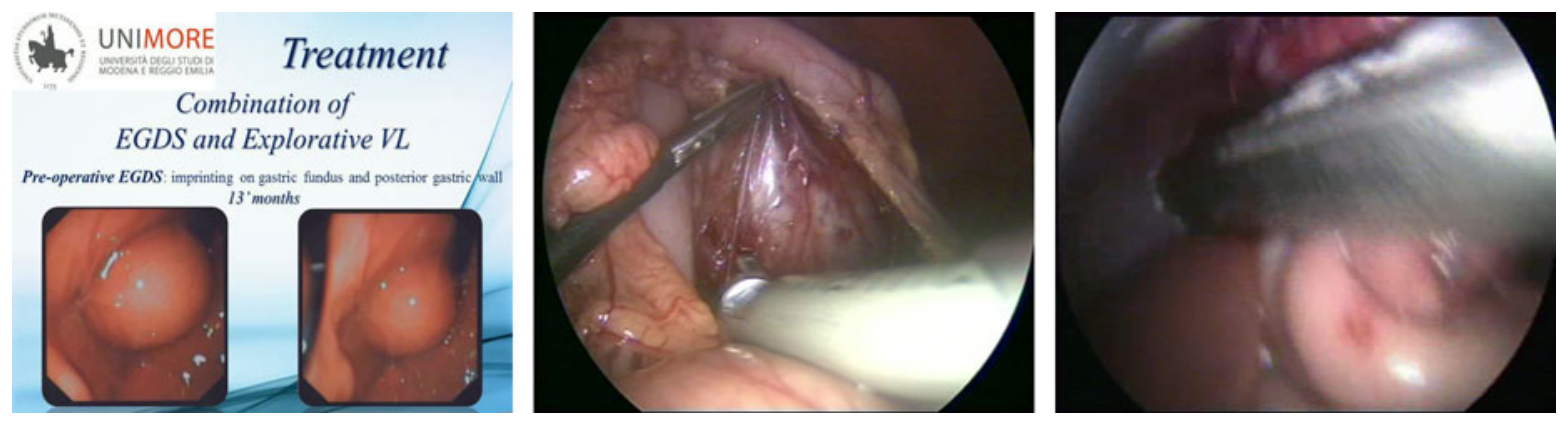

\section{Abstract}

Introduction: Gastric duplication is a rare congenital anomaly with an incidence of $4 \%-8 \%$ of all gastrointestinal duplications; enteric duplications are ectopic cystic or tubular structures with a mucous and muscular tunics and they can be in contiguity/continuity with the intestinal lumen. ${ }^{1}$ Gastric duplication is often an occasional finding, associated with aspecific symptoms for which preoperative diagnosis is not unique; differential diagnosis with other retroperitoneal diseases or mesenteric cysts can be difficult. ${ }^{1}$

Materials and Methods: We present a case of a 1-year-old child with a prenatal ultrasound (US) finding of endoabdominal cystic lesion. After birth, US scans showed an anechogenic cyst of $33 \times 28 \mathrm{~mm}$ in the left upper quadrant, between stomach, spleen, and kidney. Magnetic resonance imaging confirmed the presence of the lesion $(40 \times 34 \mathrm{~mm})$, imprinting the posterior gastric wall, the spleen, and the anterior side of left kidney. An esophagous-stomach-duodenum contrast study was also performed, showing the imprinting cyst on the great curvature close to the gastric fundus without communication with gastric lumen. On follow-up, the child underwent periodic US scans and no 
growth or ultrasonographic changes were described. At 13 months, the diagnosis was still unclear and the patient underwent explorative laparoscopy with esophagous-gastric-duodenoscopy (EGDS). The preliminary EGDS showed a $35 \mathrm{~mm}$ convexity on the posterior wall of gastric fundus with no evidence of orifice. A $5 \mathrm{~mm}$ transumbilical trocar was placed and $5 \mathrm{~mm}$ trocar in the epigastric region and $10-12 \mathrm{~mm}$ trocar in the left side were positioned. At the abdominal exploration, the cyst resulted to be in continuity with the posterior gastric wall on the superior third of the great curvature. The lesion was isolated from other tissues, but the posteromedial wall of neoformation appeared to be not dissociable and in continuity with the stomach; a complete resection of the cyst, using $45 \mathrm{~mm}$ linear stapler, was performed, including a small portion of the great gastric curvature. At the following intraoperative endoscopic control, no more evidence of irregularity of the gastric wall was seen and the suture was assured.

Results: The operative time was 140 minutes. No complications occurred and the blood loss was minimal. The patient started oral intake on the 5th postoperative day and was discharged on the 6th postoperative day. Histological examination confirmed the gastric nature of cyst. At 1 year of follow-up, no recurrences were diagnosed and the child presents in good health with a regular growth.

Conclusion: We can assume that laparoscopic surgery is the correct procedure for gastric duplication cysts, to get both definitive diagnosis and treatment, and the radical surgical excision represents the treatment of choice to avoid neoplastic degeneration of internal lining mucosa. ${ }^{2}$ Furthermore, surgical laparoscopy appears to be a feasible and safe technique. ${ }^{3}$

No competing financial interests exist.

Runtime of video: 8 mins

Keywords: gastric duplication, pediatric laparoscopy, gastric congenital anomaly

\section{Cite this video}

Barbara Catellani, Francesca Pecchini, Lorena Sorrentino, Francesca Cabry, Diego Biondini, Pier Luca Ceccarelli, Roberta Gelmini, Laparoscopic Treatment of Gastric Duplication in a Child, Videoscopy. 2017, DOI: 10.1089/vor.2017.0451.

\section{References}

1. Tanaka H, Masumoto K, Sasaki T, et al. Hypergastrinemia and a duodenal ulcer caused by gastric duplication. Surg Case Rep 2016;2:75.

2. Krishna Kumar G. Gastric duplication cyst in an infant presenting with non-bilious vomiting. Malays $J$ Med Sci 2012;19:76-78.

3. Catellani B, Pecchini F, Cabry F, et al. Case report: Duplicazione gastrica in età neonatale. Congresso Congiunto delle Società Scientifiche Italiane di Chirurgia. Italy, September 2016.

Original Publication Date: 2017 